\title{
Mutilación genital femenina. Revisión y aspectos de interés médico legal
}

\section{Female genital mutilation. Review and aspects of medico-legal interests}

\section{Resumen}

La progresiva llegada a España de personas procedentes del África subsahariana ha evidenciado la práctica de una serie de rituales ancestrales en niñas, perjudiciales para su salud, y que se engloban dentro del concepto de mutilación genital femenina (MGF).

En nuestro país estas prácticas están tipificadas como delito de lesiones y, por lo tanto, son susceptibles de plantear valoraciones médico legales. Es por ello que consideramos de especial importancia el conocimiento por parte de los profesionales de la medicina forense de una serie de aspectos generales sobre estas prácticas.

Palabras clave: Mutilación genital femenina. Ablación de clítoris. Código Penal.

\section{Abstract}

The gradual arrival in Spain of people from sub-Saharan Africa, has highlighted the practice of a series of ancient rituals in girls, harmful to their health, and which are encompassed within the concept of Female Genital Mutilation in accordance with the WHO definition.

In our country these acts are classified as a crime of injury. Therefore they are likely to raise legal medical evaluations. We consider it particularly important knowledge of these practices on the part of professionals in the forensic medicine.

Key words: Female genital mutilation. Clitoris ablation. Criminal code. Child protection measures.

\section{Introducción}

La comunidad internacional, a través de diversas agencias de Naciones Unidas y la Unión Europea, se ha pronunciado sobre ${ }^{1}$ la mutilación genital femenina (MGF) en diversos fórums, convenciones y declaraciones sobre los derechos humanos, los derechos de las mujeres y los derechos de los niños. Se trata de prácticas tradicionales, pero que constituyen una violación de los derechos humanos, en particular por su carácter discriminatorio y violento y por las consecuencias perjudiciales que conllevan para la salud de la mujer².
Se estima que alrededor de 130 millones de niñas y mujeres de todo el mundo han sido sometidas a estas prácticas y que por lo menos dos millones de niñas están en riesgo de padecer alguna de las formas de este procedimiento (la ablación se practica principalmente en niñas entre 1 y 7 años, siendo la edad más frecuente, entre 4 y 7 años, normalmente antes de la primera menstruación). La edad varía en función de los países, por ejemplo en edades más tempranas se practica en Eritrea y Mali.

En la actualidad se practica en unos cuarenta paí$\mathrm{ses}^{3}$, de los que 28 son africanos (en la región del sub-Sahara y de la parte nororiental de África), el resto son países del Próximo Oriente y Asia.

\author{
MA. Gallego \\ MI. López
}

Médico forense. Instituto de Medicina legal de Cataluña.

Correspondencia: Dra. MA. Gallego Álvarez Instituto de Medicina legal de Cataluña.

E-mail:

mariaangeles.gallego@xij. gencat.cat
Fecha de recepción: 22.ENE.2010

Fecha de aceptación: 21.FEB. 2010 
Se desconoce su origen, pero se cree que ${ }^{3}$ podría tratarse de una práctica milenaria surgida en el Antiguo Egipto, que se difundió a través de la influencia de la civilización egipcia.

Esto explicaría que la practiquen los cristianos coptos de Egipto y de Sudán, los judíos falaixa de Etiopía y las tribus africanas de culto animista.

A pesar de encontrar comunidades musulmanas que la practican, podemos afirmar que no es un precepto islámico, ni de ninguna de las principales religiones; existen zonas de influencia islámica, como pueden ser Marruecos, Argelia y Túnez que no han estado nunca vinculadas a dicha práctica. En África subsahariana, la mutilación genital femenina (MGF) se practica en toda la franja del Sahel, perdiendo fuerza a medida que nos vamos acercando a la zona ecuatorial. A partir de este punto, deja de practicarse salvo en los países de la zona de los Grandes Lagos: Kenia, Uganda, Ruanda, Burundi, Tanzania y Malawi.

Estas prácticas ancestrales han sido prohibidas por algunos gobiernos africanos, pero todavía no existe legislación al respecto. Sólo algunos países han publicado leyes sancionadoras específicas en el parlamento, como Senegal, Ghana, Mali o Mauritania. Si bien una prohibición legal es imprescindible para castigar y prevenir estas prácticas tanto en los países de origen como en los de destino de estas personas, no es suficiente si no se acompaña de otras estrategias para su prevención que puedan llegar a producir una transformación social religiosa y cultural muy amplia, dado que nos encontramos ante cuestiones de profundas diferencias culturales: diferencias entre nuestras creencias y principios básicos reconocidos en los derechos humanos universales y las creencias y tradiciones de los pueblos que la practican.

No podemos dejar de lado que los padres que realizan y promueven esta práctica creen que ésta tiene efectos beneficiosos para su hija, ya que tradicionalmente es así en su cultura de origen. Por lo tanto, si sus practicantes no entienden por qué tienen que abandonar esa práctica, es posible que la sigan llevando a cabo, a pesar de su ilegalidad.

Por otra parte si se quiere hacer realmente eficientes esas leyes, se deben elaborar planes de acción específicos que, además de prever campañas de información y sensibilización, incluyan protocolos de actuación transversales e integrales que permitan prevenir la realización de esa práctica, así como establecer medidas de protección del menor, en particular medidas cautelares, ante la detección o riesgo de estas prácticas.

Los países europeos se ven cada vez más afectados por esta práctica a través de la población inmigrante originaria de países donde la MGF constituye una práctica tradicional. Algunos inmigrantes, queriendo mantener las tradiciones de su país de origen y reforzar así su identidad cultural, practican la MGF o bien en el territorio de su país de residencia o bien en el territorio de su país de origen (o países fronterizos) aprovechando el período vacacional. En los últimos años ${ }^{4}$ ha aumentado el número de profesionales sanitarios que realizan estas prácticas en África, con lo que, en lugar de ponerles fin, tienden a legitimizarla mejorando las condiciones higiénicas en las que se realiza. Pero como recuerda la UNICEF, "tratar de que la mutilación sea segura no la convierte en algo aceptable".

La llegada a España, a partir de los años 80, de familias procedentes de países de África subsahariana, hace que se descubran realidades culturales diferentes y que se empiecen a evidenciar en nuestro país prácticas tradicionales perjudiciales para la salud de la mujer y de las menores. Por comunidades autónomas, aquellas en las que se ha detectado un mayor número de mujeres procedentes de países africanos dónde se realizan estas prácticas, son Cataluña ${ }^{5}$ (fue la primera comunidad en España dónde se detectaron casos en 1993), Andalucía, Aragón y Canarias.

En España no existe ningún protocolo de actuación preventivo a nivel estatal. A nivel autonómico, las únicas comunidades que cuentan con un protocolo de actuación o una guía de prevención son Cataluña ${ }^{6}$ y Aragón?.

En algunas otras comunidades se han elaborado guías o folletos informativos dirigidos a los profesionales, a la población de riesgo o a la población general.

El ordenamiento jurídico español tipifica estas prácticas, en cualquiera de sus formas o variantes, como un delito de lesiones.

Es por ello que consideramos de especial importancia el conocimiento por parte de los profesionales de la medicina forense de todas aquellas cuestiones relativas a las MGF que pudieran plantear una valoración médico legal en la práctica.

\section{Concepto}

Se usan diferentes términos para designar este tipo de intervenciones sobre los genitales externos femeninos, entre ellos ablación, circuncisión femenina, mutilaciones genitales femeninas, cortes genitales, sunna, cirugía genital femenina, práctica tradicional, etc. 
Pero desde las posiciones de mayor defensa de los derechos humanos se ha propuesto englobarlos en el término de Mutilación genital femenina (MGF), que cambia no sólo la orientación terminológica sino la conceptual, y se entiende que este tipo de prácticas atentan contra la integridad psico-física de las mujeres y niñas y defiende que consiste en la amputación de una parte funcional y sana del organismo femenino.

Por todo ello podemos definir las MGF como "todos aquellos procedimientos quirúrgicos que consisten en la extirpación parcial o total de los genitales externos, u otras intervenciones practicadas en los órganos genitales femeninos por razones que no son de índole médico".

\section{Razones que sustentan la mutilación genital femenina}

Las razones que sustentan la MGF son muy variadas, muchas vinculadas a creencias, con un componente social, cultural e identitario muy fuerte. Entre ellas destacan:

- Razones sociológicas: se practica como "ritual de iniciación" de las niñas en su paso a la edad adulta, o en aras de la integración y el mantenimiento de la cohesión social (la circuncisión es una "marca" para toda la vida y simboliza que su unión al grupo también lo será para toda la vida).

- Razones religiosas: la religión de algunos países así lo exige, pero así como para la circuncisión masculina es un precepto religioso, por lo tanto obligatoria, en el caso de la femenina, forma parte de la tradición, es decir, sólo tiene carácter de recomendación y no es obligatoria. Es importante resaltar que es una práctica preislámica y que el Corán no hace mención ni alusión en ningún versículo, a diferencia de la Biblia que sí lo hace para los hombres.

- Razones higiénicas y estéticas: se cree que los genitales femeninos son sucios y antiestéticos, por lo que es una forma de hacer deseable a la mujer.

- Razones psicosexuales y reproductivas: el principal motivo es controlar la sexualidad de la mujer. Se piensa que la MGF mitiga el deseo sexual y garantiza la fidelidad de la mujer. En otros casos se considera que sólo el hombre debe sentir el placer sexual, por lo que las mujeres que no han sufrido la circuncisión lo tienen muy difícil para contraer matrimonio.
Muy a menudo se cree que la MGF facilita el parto cuando los hechos demuestran lo contrario y que el clítoris puede provocar la muerte del bebé si lo roza durante el alumbramiento. También se cree que la MGF aumenta la fertilidad lo que tampoco es cierto.

Varios estudios demuestran ${ }^{8}$ que en realidad se sigue practicando la MGF sobre todo por conformidad y presión social, en particular en zonas rurales. No obstante, existen estudios que demuestran que esas consecuencias sociales (discriminatorias y excluyentes del grupo) en caso de no cumplir con la práctica no se producen necesariamente.

Lo que concluyen algunos autores es que la mayoría de razones estriban en consideraciones de género $y$, por lo tanto, en construcciones sociales que atribuyen ciertos comportamientos y funciones a las mujeres (virginidad prematrimonial, fidelidad, belleza, etc.) y que son en realidad discriminatorias.

\section{Tipología}

Si bien es cierto que el procedimiento puede variar según algunos factores como grupos étnicos y zonas geográficas, la Organización Mundial de la Salud (OMS) los ha agrupado en cuatro categorías:

- Tipo I: clitoridectomía. Eliminación del prepucio del clítoris, con o sin escisión total/parcial del clítoris. En el mundo islámico se conoce como sunna y es equivalente a lo que con frecuencia de denomina circuncisión, y que en África equiparan a la circuncisión masculina.

- Tipo Il: ablación/escisión. Extirpación de prepucio y del clítoris junto con la escisión total o parcial de labios menores, dejando intactos los labios mayores.

- Tipo III: infibulación. Extirpación del clítoris y de la totalidad de los labios mayores y menores. Posteriormente se suturan ambos lados de la vulva dejando un pequeño orificio que permite la salida de la orina y del flujo menstrual.

- Tipo IV: otros. Todos aquellos procedimientos que impliquen la extirpación parcial o total de los genitales femeninos. Se han descrito prácticas de severidad variable como:

- Pinchado, anillamiento o incisión del clítoris y/o de los labios vaginales, cosido o estrechamiento del clítoris y/o de los labios vaginales y cauterización mediante el quemado del clítoris y del tejido que lo rodea. 
- También se encuentran frecuentemente mujeres a las que se les ha practicado otro tipo de mutilaciones como la introducción de hierbas corrosivas en la vagina para provocar el sangrado o su estrechamiento, el raspado del tejido que rodea el orificio vaginal, cortes en el interior de la vagina... que si bien no implican la escisión total o parcial de los genitales femeninos, por su repercusión sobre los mismos consideramos incluirlas en este punto.

Según datos de Amnistía Internacional, el 15\% de las mutilaciones que se practican en África son infibulaciones, el resto son clitoridectomías o distintos tipos de escisión.

En España, por el origen de los inmigrantes, las que se han detectado son el tipo I y II, practicándose a edades entre los 4 y 6 años, aunque puede variar, pero normalmente antes de la primera menstruación.

\section{Consecuencias para la salud de la mujer}

Es fácil deducir que la práctica de estas técnicas tiene unas consecuencias a corto y largo plazo para la salud física y psicológica de la mujer, sobre todo teniendo en cuenta que generalmente se realizan con material no esterilizado (se describe la práctica con un cuchillo, una hoja de afeitar, un pedazo de vidrio... y la utilización para las suturas de fibras vegetales, alambre, hilo de pescar...), en condiciones sépticas (en camastros, sobre el suelo) y por personal no cualificado (comadronas tradicionales sin titulación profesional, una persona anciana del grupo, barberos, curanderos) que no están formados en la asepsia ni en la protección de enfermedades.

En la mayoría de los casos, el grado de mutilación condiciona el tipo de complicación que se puede presentar; siempre son más graves en el caso de mujeres infibuladas. De forma general se clasifican en:

- Complicaciones inmediatas (con posible riesgo vital): la región vulvar es rica en irrigación e inervación, por lo que la ablación del clítoris y de los labios produce un intenso dolor asociado a miedo y angustia. El intenso dolor dificulta la micción produciendo una retención urinaria refleja. La hemorragia en los casos más graves puede llevar a la muerte de la víctima por shock hipovolémico, e incluso el shock neurógeno (debido al dolor intenso) o séptico pueden ser los responsables del óbito.
- Complicaciones tardías o diferidas (que son las que encontraremos frecuentemente en nuestro ámbito):

- Generales:

- Dado que estas prácticas suelen practicarse con medios poco higiénicos, es frecuente que se produzcan infecciones subagudas (vulvares, urinarias, e incluso puede aparecer tétanos, hepatitis o infección por HIV).

- La hemorragia que se suele producir como consecuencia de la mutilación, añadida a problemas de alimentación deficiente y a las anemias hereditarias, puede provocar una anemia severa.

- Consecuencias psicológicas a medio y largo plazo provocadas por los sentimientos contradictorios producidos por las diferencias de valores entre la sociedad en la que viven y la cultura a la que pertenecen, que provocan miedo a ser rechazadas por su propia gente si no se someten a la ablación; en su cultura, una mujer que no se haya sometido a la MGF difícilmente podrá casarse y no merece ningún respeto (sentimientos de humillación y vergüenza). Esto puede derivar en angustia y depresión. Además se ha identificado un síndrome específico: "genitally focused ansiexety depression", caracterizado por una constante preocupación sobre el estado de los genitales y el pánico a la infertilidad.

- Complicaciones obstétricas, urinarias, sexuales y ginecológicas:

- Complicaciones obstétricas: las complicaciones en el momento del parto dependen del grado de mutilación.

En el tipo I son poco frecuentes, aunque son posibles los desgarros de la cicatriz. En el tipo II y III, dado que hay extirpación de tejidos y formación de cicatrices de tipo queloide y de quistes dermoides, la zona perineal es poco elástica y presenta dificultades para dilatarse durante el período expulsivo. Las cicatrices y los quistes desfiguran la anatomía habitual y esto dificulta el sondaje vesical en el momento del parto, favoreciendo retención de orina en el posparto e infecciones urinarias y hemorragias por atonía del útero. 
- Complicaciones sexuales: cualquier tipo de MGF interfiere de alguna forma en la respuesta sexual, aunque no suprima necesariamente la posibilidad de placer y orgasmo, ya que también interfieren otros factores. Puede aparecer dispareunia, disminución del deseo sexual, anorgasmia y modificación de la sensibilidad sexual.

- Complicaciones genitourinarias: infecciones urinarias de repetición secundarias al lento y doloroso vaciamiento de la vejiga que puede tardar hasta quince minutos, por lo que en ocasiones se resisten a beber líquidos. Retenciones e incontinencia urinaria si la uretra queda lesionada.

Dismenorrea o regla dolorosa. Hematocolpos: la menstruación puede durar diez días o más, el cierre de la vulva provoca retención de la sangre, por lo que es habitual el mal olor. Infertilidad secundaria a infecciones del aparato reproductor. Infecciones vaginales y uterinas relativamente frecuentes.

\section{Marco legal}

Las sucesivas campañas de sensibilización con respecto a la MGF que se están llevando a cabo por numerosas organizaciones que luchan por los derechos humanos de mujeres y niños han llevado a que algunos países, que tenían estas prácticas legalizadas, decidan prohibirlas y dictar leyes sancionadoras al respecto.

En Europa ${ }^{2}$, varios Estados han aprobado leyes específicas (Noruega, Suecia, Reino Unido) o han modificado su legislación (Austria, Bélgica, Dinamarca y España) para tipificar la MGF como delito. En otros queda legalmente prohibida bajo la aplicación de leyes penales generales y suele ser asimilada a un atentado contra la integridad física y moral de la persona (Alemania, Finlandia, Francia, Grecia, Italia, Países Bajos y Suiza, entre otros).

En nuestro país la MGF, en cualquiera de sus tipos, está contemplada dentro del delito de lesiones del Código Penal actualmente vigente (ley 10/1995 modificado por la ley orgánica 11/2003), en los artículos ${ }^{9}$ :

- Artículo 149.2: "El que causare a otro una mutilación genital en cualquiera de sus manifestaciones será castigado con la pena de prisión de 6 a 12 años. Si la víctima fuera menor o incapaz será aplicada la pena de inhabilitación especial para el ejercicio de la patria potestad, tutela, curatela, guarda o acogimiento por tiempo de 4 a 10 años, si el juez lo estima adecuado para el interés del menor incapaz".

- Artículo 151: castiga la conspiración y la proposición para cometer los delitos de lesiones.

Podría considerarse que si en España los padres llegaran al acuerdo de realizar la mutilación a su hija (sea en España o en el extranjero), dicho acuerdo entraría en la figura de la conspiración para cometer un delito de lesiones, mientras que en el supuesto de que uno de los progenitores lo decidiese y le propusiese al otro llevarlo a cabo, esta situación entraría dentro de la figura de la proposición para cometer delito de lesiones.

La aprobación ${ }^{10}$ de la Ley orgánica 3/2005 de 8 de julio de la jefatura de Estado modificó la Ley Orgánica 6/1985 para permitir la persecución de este delito aunque haya sido cometido fuera de España, siempre que los autores o la persona afectada se encuentren en el territorio español. Esto representa un avance en el marco del llamado principio de "universalidad" de la justicia.

En la aplicación práctica de la legislación, cuando haya detección de un caso de MGF, ya sea en el ámbito sanitario, social, policial o educativo, existe por parte de estos colectivos la obligación de ponerlo en comunicación judicial, por estar englobado en nuestro ordenamiento jurídico dentro del delito de lesiones.

Lo que no prevee la legislación actualmente vigente son las medidas cautelares en caso de sospecha o riesgo de que pueda ocurrir una MGF. Aún así, hay diferentes protocolos ${ }^{1,3,11}$ que establecen la recomendación de ponerlo en comunicación del Ministerio Fiscal a efectos de investigación y posterior adopción de medidas cautelares para la prevención (y que en la práctica se han tomado en España en algunas ocasiones). Entre los factores/indicadores de riesgo contemplados ${ }^{2}$, destacan:

- Familia procedente de un país dónde se suele practicar la MGF.

- Madre u otro miembro de la familia que ha sufrido dicha práctica.

- Edad de la menor.

- Proximidad de un viaje al país de origen.

- Postura a favor de los progenitores respecto a la MGF. 
Dentro de las medidas cautelares destacan:

- Citación de los padres para conocer sus intenciones e informarles sobre las consecuencias de la MGF tanto desde el punto de vista de la salud como desde un punto de vista legal.

- Acordar la presentación periódica de la menor a un médico (pediatra, forense) a efectos de control. En relación con esta medida, la asociación catalana de matronas ${ }^{1}$ también propone la realización de exploraciones genitales periódicas para las niñas en situación de riesgo y sugiere la periodicidad siguiente: una primera vista a la recién nacida, luego al año, a los 2, 4, 6, 8 y 10 años, así como cualquier examen dentro del protocolo habitual previsto para los niños, y los vinculados a la realización de un viaje.

- Requerirlos para que se abstengan de sacar a la menor fuera del territorio español sin comunicarlo al juzgado con la antelación que se determine.

- Si los padres proyectan un viaje a su país de origen, ordenar un examen médico antes y después del viaje. En caso de actitud sospechosa prohibir a los padres que trasladen a la menor fuera del territorio español; retirarles el pasaporte o prohibir su expedición.

Cabe destacar que el Instituto Catalán de la Salud usa un documento médico que tiene doble utilidad, por un lado sirve de certificado médico para garantizar que los genitales de la menor están íntegros y no presentan lesiones, y por otro lado sirve de carta de compromiso de los padres (o tutor) en la que declaran haber sido informados sobre los riesgos de la MGF y del marco legal en España y se comprometen a cuidar de la niña y evitar su mutilación genital, y a volver con ella a su regreso, para su control.

\section{Intervención del médico forense}

La intervención del médico forense en relación a este tipo de prácticas aparece en el momento en que se comunica al juzgado la sospecha o la detección de un caso de MGF. La frecuencia de estas periciales está directamente relacionada con las zonas que concentran mayor número de inmigrantes procedentes de países donde se llevan a cabo estas prácticas.

Atendiendo a todo lo anteriormente expuesto, consideramos los siguientes ámbitos de actuación posibles:
- Dentro de las medidas cautelares en caso de riesgo de proceder a esta práctica con una menor, destacan las revisiones médicas periódicas, a efectos de detectar cualquier posible lesión, que realizaría el médico asistencial pero que también podrían ser realizadas por el médico forense.

- Cuando se pone en conocimiento la existencia de una menor a la que se le ha realizado dicha práctica, el médico forense podría intervenir fundamentalmente a través del reconocimiento y exploración física y psicológica de la menor emitiendo el preceptivo informe de valoración del daño corporal, en el que destaca por su importancia no sólo el tipo de ablación sufrida sino las secuelas resultantes de dicha práctica, incluyendo dentro de las mismas las posibles repercusiones psicológicas que pudieran derivarse en la menor explorada.

- Dado el impacto que se produce en la salud física de la menor, y atendiendo a las condiciones en que se practica, uno de los riesgos fundamentales es que se produzca la muerte de ésta durante o posterior a la práctica. De producirse esta muerte sería tributaria de autopsia judicial. En estos casos, la causa de la muerte debe ir íntimamente ligada a establecer el nexo causal entre ésta y las prácticas alegadas.

Dentro de las causas de muerte probables destacan las muertes relacionadas con las hemorragias masivas y shock séptico en caso de muerte inmediata, y las secundarias a infecciones u otras complicaciones en caso de muertes diferidas.

- Podría solicitarse por parte del juzgador o de la parte, la valoración psicológica de los padres a efectos de dictaminar sobre su competencia legal.

Consideramos este extremo de difícil valoración ya que la competencia legal específica puede estar alterada atendiendo a la percepción favorable y beneficiosa que tienen estas familias de dichas prácticas (rituales arraigados en su grupo con presión social importante por parte del mismo) y a la ausencia de conocimiento del perjuicio físico y psicológico que crea en las menores.

- Cabe considerar un último extremo en relación a las probables pericias solicitadas en relación a las MGF. Atendiendo a que se conoce de familias que han pedido la ablación a hospitales públicos -extremo denunciado por el defensor del pueblo- y que en una mujer embarazada 
que haya sufrido una infibulación que se ha de reparar previamente al parto, no se puede reconstruir posteriormente, no hay que descartar la posibilidad de una posible petición de valoración de la praxis médica en estos supuestos.

\section{Conclusión}

Nos encontramos ante una realidad muy compleja, que merece un enfoque integral por parte de las administraciones implicadas. Es un problema de salud que trasciende el marco puramente asistencial y judicial, en el que confluyen la vulneración de los derechos humanos, la necesidad de un abordaje transcultural de cuestiones íntimamente ligadas a la identidad de las personas, y el compromiso de evitar unas prácticas tradicionales que implican el trato discriminatorio, violento, degradante y doloroso hacia las mujeres.

Es por ello que el uso del derecho penal como mecanismo sancionador no puede desvincularse de una adecuada labor de información y prevención a través de organismos como es la escuela, los servicios sociales, profesionales sanitarios, mediadores interculturales y educadores para llegar entre todos a una resolución efectiva del problema.

\section{Agradecimientos}

A los revisores de la revista por su aportación crítica constructiva.

A Celia y Mireia de la Bibilioteca del Instituto de Medicina Legal de Cataluña.

\section{Bibliografía}

1. Associació Catalana de Llevadores. Mutilación genital femenina: prevención y atención. Barcelona: Asociación catalana de matronas. 2004 (última actualización 2007).

2. Lucas B. Prevención de la ablación o mutilación genital femenina en España: Planes de acción y medias de protección de menores complementos necesarios a la prohibición legal. Cuadernos electrónicos de filosofía del derecho 2008;17.

3. Comisión de trabajo sobre la violencia doméstica de Cataluña. Protocolo de prevención sobre la mutilación genital femenina. Generalitat de Cataluña 2004

4. Martín Espildora N. Atención de menores inmigrantes en la consulta: entre la diferencia cultural y el delito. Un caso de ablación de clítoris. Aten. Primaria 2005;36(7):397-400.

5. Kaplan Marcusan A, Torán Monserrat P, Bedoya Muriel MH, Bermúdez Anderson K, Moreno Navarro J, Bolíbar Ribas B. Las Mutilaciones gentiales femeni- nas: reflexiones para una intervención desde la atención primaria. Aten. primaria 2006;38(2):122-6.

6. Comisión de trabajo sobre la violencia doméstica. Protocolo de prevención de la mutilación genital femenina en la demarcación de Girona. Generalitat de Catalunya, 2002 (última actualización en 2006).

7. Comisión Interdisciplinar para la prevención de la mutilación genital femenina. Guía de prevención de la mutilación genital femenina. Gobierno de Aragón, 2004.

8. Lucas B. Aproximación antropológica a la práctica de la ablación o Mutilación Genital Femenina. Cuadernos electrónicos de filosofía del derecho $2008 ; 17$

9. Ley Orgánica 10/1995 del Código Penal.

10. Ley orgánica $3 / 2005$, de 8 de julio, de modificación de la Ley Orgánica 6/1985, de 1 de julio, del Poder Judicial, para perseguir extraterritorialmente la práctica de la mutilación genital femenina.

11. Jornada sobre la prevención de la mutilación genital femenina. Mataró 2005. 